\title{
Aberration-Corrected STEM
}

\author{
N.J. Bacon, G.J. Corbin, N. Dellby, P. Hrncirik, O.L. Krivanek, M.F. Murfitt, C.S. Own and Z.S. \\ Szilagyi
}

Nion Co., $11028^{\text {th }}$ St., Kirkland, WA 98033, USA

A scanning transmission electron microscope's (STEM's) performance is primarily determined by seven factors:

1) electron source: brightness, energy spread and stability; monochromator performance

2) probe-forming optics: aberration characteristics, stability, flexibility

3) sample stage: vibration immunity, motion precision \& reproducibility, freedom from drift

4) detector-coupling optics: aberration characteristics, stability, flexibility

5) detectors: coupling efficiency, DQE, speed, flexibility

6) overall vacuum: freedom from contamination and sample etching

7) operating software: flexibility, convenience, user friendliness

With the Nion UltraSTEM [1], the Nion design team has made major progress in all seven areas. Here we review our progress in areas 1-3 and 6-7; progress in areas 4 and 5 is described in another contribution to these proceedings [2].

Electron source: Our $200 \mathrm{kV}$ CFEG source is now operating largely as expected. Its design [3] concentrates on several aspects not properly addressed previously. The ultimate brightness of CFEG sources is mainly determined by the emitter workfunction and stochastic Coulomb interactions affecting electrons traveling between the emitter and the principal beam-defining aperture. Coulomb effects increase rapidly with the total emitted current and the highest brightness for a source is achieved when a further increase in total current is negated by Coulomb source spread. This implies that the electrons should be accelerated away from the tip as fast as possible. Maximizing the stability requires careful UHV design, bakeability to high temperatures, and efficient pumping close to the source. Our design and the results it has produced will be described at the meeting.

We have also designed (but not yet built) a monochromator for the source section of the column [4]. Our monochromator differs in major ways from present-day monochromator designs: it performs the monochromation on the electron beam at its full energy, its energy dispersion is variable, it ties the monochromator, the system's energy-loss spectrometer and the gun HT supply together in a stability-enhancing scheme, and it is able to correct the chromatic aberration of the whole probeforming column. We estimate that it will be able to attain $10-30 \mathrm{meV}$ energy resolution (at $100 \mathrm{kV}$ primary voltage), and that its $\mathrm{C}_{\mathrm{c}}$ correction will significantly improve the attainable spatial resolution of the STEM, especially at primary voltages of $100 \mathrm{kV}$ and lower.

The monochromator uses an aberration correction principle that would have pleased Otto Scherzer: correction of $\mathrm{C}_{\mathrm{c}}$ by sextupole lenses acting on an energy-dispersed beam. To our knowledge, this type of correction has never been tried in electron microscopy before, even though it is used in practically all accelerator rings. 
Probe-forming optics: The probe-forming column features 3-4 condenser lenses (4-5 with the monochromator), a $\mathrm{C}_{3} / \mathrm{C}_{5}$ aberration corrector, a flexible scanning system, a beam blanker with $<10$ $\mu$ s blanking/unblanking time, and an objective lens optimized for lowest $\mathrm{C}_{\mathrm{c}}$ rather than lowest $\mathrm{C}_{\mathrm{s}}$. It is able to switch between standard high-resolution imaging $(\sim 100$ pA beam current, probe size $<1$ $\AA$ at $100 \mathrm{kV})$, high-current imaging/spectroscopy $(0.5-1 \mathrm{nA}$ current in a $\sim 1.5 \AA$ probe), diffractionlimited nanoprobe mode $(\sim 50 \mathrm{pA}$ current in a diffraction-limited probe of $\sim 1 \mathrm{mr}$ total convergence, useful for phase identification via nanodiffraction) and diffraction-limited parallel probe mode $(\sim 50$ $\mathrm{pA}$ of current in a diffraction-limited probe of $\sim 0.1 \mathrm{mr}$ total convergence, useful for precision lattice strain measurements), all with the same illumination aperture and typically returning to the same sample area with precision better than $10 \mathrm{~nm}$ on switching between the modes.

Sample stage: Our friction-free design that compensates for thermal expansion is able to move the sample mechanically with a smallest increment of $\sim 1 \mathrm{~nm}$ in $\mathrm{X}$ and $\mathrm{Y}$, and about $5 \mathrm{~nm}$ in Z. Its lowest resonant frequency of $1200 \mathrm{~Hz}$ makes it uncommonly immune to external disturbances, and its stability has been measured as high as $1 \AA$ total drift in 3 minutes. Its ball-bearings-only doubletilt cartridge leads to reproducible mechanical tilts as small as 0.03 degrees.

Overall vacuum: Only metal seals are used in the column up to the detector section, where Viton O-rings are permitted. The vacuum levels are: gun $<<1 \times 10^{-10}$ torr, column from gun to projectors $\sim 1 \times 10^{-9}$ torr, detector section $\sim 2 \times 10^{-7}$ torr. The whole microscope and batches of samples (before their introduction into the microscope) can be baked at $140^{\circ} \mathrm{C}$. The result is that there is no contamination or sample etching on nearly all standard materials science type samples.

The sample stage is pumped by a $30 \mathrm{l} / \mathrm{s}$ ion pump, yet it typically reaches into the high $10^{-10}$ torr range (in the latest version of the microscope). Adding larger-capacity pumping by incorporating a Ti sublimation pump directly in the sample chamber should allow it to reach the $10^{-11}$ torr range and make it suitable for surface-science experiments.

Operating software: As with many software projects, software development at Nion is based on Kaizen principles [5]: areas needing improvement are continuously evaluated, and the software is updated at regular intervals. In the latest software release, bringing up the microscope's high voltage and turning the emission on are achieved by two clicks of the computer mouse, and the computer-controlled sample exchange is activated by a single click. The sample is moved and tilted by Mapquest-like "click-to-shift" and "click-to-tilt", whereby the sample location (or axis) the user clicks on is automatically moved (tilted) into the center of the viewed area. Aberration tuning and voltage and aperture centering have been completely automated. At the same time, access to lowlevel controls such as individual lens currents or deflector settings is allowed for advanced users.

Several of the microscope's unique design features will be described in more detail, and examples of practical applications will be presented.

[1] O.L. Krivanek et al., Ultramicroscopy 108 (2008) 179.

[2] O.L. Krivanek et al. (2009) these proceedings.

[3] N.J. Bacon et al., to be published.

[4] O.L. Krivanek et al., Phil Trans Roy Soc. (2009) in print.

[5] http://en.wikipedia.org/wiki/Kaizen 